\title{
NON COMMUTATIVITY OF SELF HOMOTOPY GROUPS
}

\author{
Hideaki Ōshima and NobUaKi Yagita
}

\begin{abstract}
We study non-commutativity of the self homotopy groups of Lie groups.
\end{abstract}

\section{Introduction}

Let $G$ be a connected Lie group and $\mu: G \times G \rightarrow G$ the multiplication of $G$. For any space $A$ with a base point, the based homotopy set $[A, G]$ becomes a group with respect to the binary operation $\mu_{*}:[A, G] \times[A, G]=[A, G \times G] \rightarrow$ $[A, G]$. Even if $A$ is a simple space, it is difficult to calculate the group $[A, G]$. A general result was given by Whitehead (p. 464 of [17]):

$$
\operatorname{nil}[A, G] \leq \text { cat } A,
$$

where nil and cat denote the nilpotency class and the Lusternik-Schnirelmann category with $\operatorname{cat}(*)=0$, respectively. We study the special case $A=G$. In [9], [12], [13], the group $[G, G]$ has been calculated for $G=S U(3), S p(2), G_{2}$. It shows that nil[G,G] equals 2 if $G=S U(3), S p(2)$ and 3 if $G=G_{2}$. This supports the following conjectures which were proposed in [13] by the first author.

Conjecture 1.1. If $G$ is simple, then $\operatorname{nil}[G, G] \geq \operatorname{rank} G$.

Conjecture 1.2. If $G$ is simple and rank $G \geq 2$, then $\operatorname{nil}[G, G] \geq 2$, that is, $[G, G]$ is not commutative.

If 1.1 is affirmative, then so is 1.2. Notice that two conjectures are false in general without the assumption of simpleness of $G([13])$.

The purpose of this note is to prove the following which supports the above conjectures.

1991 Mathematics Subject Classification: 55Q05.

Key words and phrases: Lie group, self map, nilpotency class, Morava K-theory.

Received December 6, 1999. 
THEOREM 1.3. (1) nil $[S U(4), S U(4)]=3$.

(2) $\operatorname{nil}[G, G] \geq 2$ if $G=S U(5), S U(6), S p(3)$.

(3) $\operatorname{nil}[G, G] \geq 3$ if $G=\operatorname{Spin}(7), \operatorname{Spin}(8), E_{6}, F_{4}$.

(4) $\operatorname{nil}\left[E_{8}, E_{8}\right] \geq 5$.

We study $\operatorname{Spin}(7), \operatorname{Spin}(8), E_{6}, E_{8}, F_{4}$ in $\S 2, S U(4)$ in $\S 3$, and $S U(5), S U(6)$, $S p(3)$ in $\S 4$.

We do not distinguish notationally between a map and its homotopy class.

\section{2. $\bmod p$ non commutativity}

For any space $Y$ with a base point, we denote by $d_{n}$ the diagonal maps $Y \rightarrow$ $\underbrace{Y \times \cdots \times Y}_{n}$ and $Y \rightarrow \underbrace{Y \wedge \cdots \wedge Y}_{n}$.

Let $X$ be a connected homotopy associative CW Hopf space. The commutator map $c_{2}: X \times X \rightarrow X$ is the composite of

$$
\begin{aligned}
X \times X \stackrel{d_{2} \times d_{2}}{\longrightarrow} X \times X \times X \times X \stackrel{1 \times t w \times 1}{\longrightarrow} X \times X \times X \times X \\
\stackrel{1 \times 1 \times \sigma \times \sigma}{\longrightarrow} X \times X \times X \times X \stackrel{\mu \times \mu}{\longrightarrow} X \times X \stackrel{\mu}{\longrightarrow} X
\end{aligned}
$$

where $t w$ is the twisting map, $\sigma$ is the inverse and $\mu$ is the multiplication of $X$. Inductively we define $c_{n}=c_{2} \circ\left(1 \times c_{n-1}\right): \underbrace{X \times \cdots \times X} \rightarrow X$ for $n \geq 3$. Of course, when $X$ is a topological group, $c_{2}(x, y)=x y x^{-1} y^{-1}$ and $c_{n}$ can be seen as a map $\underbrace{X \wedge \cdots \wedge}_{n} \rightarrow X$ for $n \geq 2$. Given $f, g \in[Y, X]$, its commutator $[f, g] \in$ $[Y, X]$ is represented by the map

$$
Y \stackrel{d_{2}}{\longrightarrow} Y \times Y \stackrel{f \times g}{\longrightarrow} X \times X \stackrel{c_{2}}{\longrightarrow} X .
$$

Let $p$ be an odd prime and $h_{*}(-)$ the $\bmod p$ ordinary homology $H_{*}\left(-; \boldsymbol{Z}_{p}\right)$ or the Morava K-theory $K(n)_{*}(-)$ with the coefficient $K(n)_{*}=\boldsymbol{Z}_{p}\left[v_{n}, v_{n}^{-1}\right],\left|v_{n}\right|=$ $2\left(p^{n}-1\right)$. We assume that $H_{i}\left(X ; \boldsymbol{Z}_{p}\right)$ is finite dimensional for every $i$. Thus $h_{*}(X)$ is a Hopf algebra with the multiplication $\mu_{*}$ and the comultiplication $d_{2 *}$. Hence $h_{*}(X)$ is cocommutative but, in general, not commutative. Given $x \in$ $h_{s}(X)$ and $y \in h_{t}(X)$, we define

$$
[x, y]=x y-(-1)^{s t} y x \in h_{s+t}(X) .
$$

By direct calculation, we have

Lemma 2.1 ([14], [19]). If $x_{1}, \ldots, x_{n} \in h_{*}(X)$ are primitive $(n \geq 2)$, then

$$
c_{n *}\left(x_{1} \otimes \cdots \otimes x_{n}\right)=\left[x_{1},\left[x_{2}, \ldots[x_{n-1}, x_{n} \underbrace{] \ldots]]}_{n-1}\right.\right.
$$

and it is primitive. 
By the Borel theorem, the $\bmod p$ cohomology $H^{*}\left(X ; \boldsymbol{Z}_{p}\right)$ is a tensor product of truncated polynomial algebras and exterior algebras generated by even and odd dimensional elements respectively. In particular, mod $p$-cohomology of exceptional Lie groups have form

$$
H^{*}\left(X ; \boldsymbol{Z}_{p}\right)=\bigotimes_{i, j} \boldsymbol{Z}_{p}\left[y_{i}\right] /\left(y_{i}^{p}\right) \otimes \Lambda\left(x_{j}\right),
$$

where $\left|y_{i}\right|$ is even and $\left|x_{j}\right|$ is odd. The $\bmod p$ homology is the dual of the cohomology and is additively isomorphic to the cohomology. Let us denote by $z_{j}$ (resp. $\left.y_{i}\right)$ the dual of $x_{j}$ (resp. $y_{i}$ ). We have

TheOREM 2.2 ([6], [7]). Let $G$ be an exceptional Lie group having p-torsion in homology. Then for some $n$ with $2 \leq n \leq 3$, we have

(1) $K(n)_{*}(G) \cong K(n)_{*} \otimes H_{*}\left(G ; \mathrm{Z}_{p}\right)$.

(2) For each $z_{j} \neq z_{3}$, there is $y_{i}$ such that $c_{2 *}\left(y_{i} \otimes z_{j}\right) \neq 0$ in $K(n)_{*}(G)$.

Notice that $G$ of the above theorem is one of $F_{4}, E_{6}, E_{7}, E_{8}$ for $p=3$ and $E_{8}$ for $p=5$. In these cases, all $y_{i}$ and $z_{j}$ are primitive. Except the case $G=E_{8}$ for $p=3$, Theorem 2.2 holds for $n=2$.

By definition, we easily have

$$
\text { Lemma 2.3. } \quad c_{2 *}(1 \otimes 1)=1 \text { and } c_{2 *}(\alpha \otimes 1)=c_{2 *}(1 \otimes \alpha)=0 \text { for } \alpha \in \tilde{h}_{*}(X) .
$$

Localization technique works for our purpose. For any prime number $p$ (including the case $p=2$ ), let $X_{(p)}$ be the $p$-localization of $X$. Then $\left[X_{(p)}, X_{(p)}\right]$ $\cong[X, X]_{(p)}$ and

$$
\operatorname{nil}[X, X]=\max _{p}\left\{\operatorname{nil}\left[X_{(p)}, X_{(p)}\right]\right\} .
$$

Now we consider the concrete cases. Harper [2], Harris [3] and Wilkerson [18] showed that there are decompositions of $\bmod p$ spaces (not as $\mathrm{H}$-spaces):

$$
F_{4} \simeq_{3} F_{4}^{\prime} \times F_{4}^{\prime \prime}, \quad E_{6} \simeq_{3} F_{4} \times\left(E_{6} / F_{4}\right), \quad E_{8} \simeq_{5} E_{8}^{\prime} \times E_{8}^{\prime \prime}
$$

where

$$
\begin{gathered}
H^{*}\left(F_{4}^{\prime} ; \mathrm{Z}_{3}\right)=\mathrm{Z}_{3}\left[y_{8}\right] /\left(y_{8}^{3}\right) \otimes \Lambda\left(x_{3}, x_{7}\right), \quad H^{*}\left(F_{4}^{\prime \prime} ; \mathrm{Z}_{3}\right)=\Lambda\left(x_{11}, x_{15}\right), \\
H^{*}\left(E_{8}^{\prime} ; \mathrm{Z}_{5}\right)=\mathrm{Z}_{5}\left[y_{12}\right] /\left(y_{12}^{5}\right) \otimes \Lambda\left(x_{3}, x_{11}, x_{27}, x_{35}\right), \\
H^{*}\left(E_{8}^{\prime \prime} ; \mathrm{Z}_{5}\right)=\Lambda\left(x_{15}, x_{23}, x_{39}, x_{47}\right) .
\end{gathered}
$$

The action $\left[y_{8},-\right]$ (resp. $\left.\left[y_{12},-\right]\right)$ in $K(2)_{*}(G)$ for $(G, p)=\left(F_{4}, 3\right)\left(\operatorname{resp} .\left(E_{8}, 5\right)\right)$ is given as follows [6]:

$$
\begin{gathered}
z_{3} \rightarrow z_{11} \rightarrow-v_{2} z_{3}, \quad z_{7} \rightarrow z_{15} \rightarrow-v_{2} z_{7} \\
\text { (resp. } \left.z_{3} \rightarrow z_{15} \rightarrow z_{27} \rightarrow z_{39} \rightarrow-v_{2} z_{3}, \quad z_{11} \rightarrow z_{23} \rightarrow z_{35} \rightarrow z_{47} \rightarrow-v_{2} z_{11}\right) .
\end{gathered}
$$


Corollary 2.4. If $(G, p)$ is $\left(F_{4}, 3\right),\left(E_{6}, 3\right)$ or $\left(E_{8}, 5\right)$, then $\operatorname{nil}[G, G] \geq p$.

Proof. Under the condition, we have $y^{p}=-v_{2} y$ in $K(2)_{*}(G)$ by (1.4) of [20]. As is well-known,

$$
\operatorname{ad}^{k}(y)(z):=\left[y,\left[y, \ldots[y, z \underbrace{] \ldots]]}_{k}=\sum_{l=0}^{k}\left(\begin{array}{l}
k \\
l
\end{array}\right)(-1)^{l} y^{k-l} z y^{l} .\right.\right.
$$

In particular $\operatorname{ad}^{p}(y)(z)=y^{p} z-z y^{p}=-v_{2}[y, z] \neq 0$, whence $\operatorname{ad}^{p-1}(y)(z) \neq 0$.

Let $f$ be the composite of

$$
F_{4(3)} \stackrel{\text { proj }}{\longrightarrow} F_{4(3)}^{\prime} \stackrel{\subset}{\longrightarrow} F_{4(3)} .
$$

Then

$$
f_{*}\left(y_{8}\right)=y_{8} \text { and } f_{*}\left(z_{15}\right)=0 .
$$

By direct computation of the diagonal map, we have

$$
d_{3 *}\left(y_{8}^{2} z_{15}\right)=2 y_{8} \otimes y_{8} \otimes z_{15}+a,
$$

where $a=\sum a_{1} \otimes a_{2} \otimes a_{3}$ such that $a_{i}=z_{15}$ for some $i \leq 2$ or $a_{i}=1$ for one or two $i$ 's and $a_{j} \in \widetilde{K(2)_{*}}\left(F_{4(3)}\right)\left(=\widetilde{K(2)_{*}}\left(F_{4}\right)\right)$ for all $j$ with $a_{j} \neq 1$. Since $c_{3 *}(f \times f \times i d)_{*}(a)=0$ by 2.3 and (2.2), it follows from 2.1 that we have

$$
\begin{aligned}
{[f,[f, i d]]_{*}\left(y_{8}^{2} z_{15}\right) } & =c_{3 *}(f \times f \times i d)_{*} d_{3 *}\left(y_{8}^{2} z_{15}\right)=2 \operatorname{ad}^{2}\left(y_{8}\right)\left(z_{15}\right) \\
& =-\operatorname{ad}^{2}\left(y_{8}\right)\left(z_{15}\right) \neq 0
\end{aligned}
$$

in $K(2)_{*}\left(F_{4}\right)$. Hence $[f,[f, i d]] \neq 0$ and $\operatorname{nil}\left[F_{4(3)}, F_{4(3)}\right] \geq 3$ so that $\operatorname{nil}\left[F_{4}, F_{4}\right] \geq 3$ by (2.1).

Let $\tilde{f}$ be the composite of

$$
E_{6(3)} \stackrel{\text { proj }}{\longrightarrow} F_{4(3)} \stackrel{f}{\longrightarrow} F_{4(3)} \stackrel{i}{\subset} E_{6(3)} .
$$

We have $i^{*}[\tilde{f},[\tilde{f}, i d]]=i_{*}[f,[f, i d]] \neq 0$ in $\left[F_{4(3)}, E_{6(3)}\right]$, because $i_{*}:\left[F_{4(3)}, F_{4(3)}\right] \rightarrow$ $\left[F_{4(3)}, E_{6(3)}\right]$ is injective. Hence $[\tilde{f},[\tilde{f}, i d]] \neq 0$ in $K(2)_{*}\left(E_{6(3)}\right)$ and $\operatorname{nil}\left[E_{6(3)}, E_{6(3)}\right]$ $\geq 3$ so that nil $\left[E_{6}, E_{6}\right] \geq 3$ by (2.1).

Let $g$ be the composite of

$$
E_{8(5)} \stackrel{\text { proj }}{\longrightarrow} E_{8(5)}^{\prime} \stackrel{\subset}{\longrightarrow} E_{8(5)} .
$$

Then

$$
g_{*}\left(y_{12}\right)=y_{12} \quad \text { and } \quad g_{*}\left(z_{15}\right)=0 \text {. }
$$

We have

$$
d_{5 *}\left(y_{12}^{4} z_{15}\right)=(5-1) ! y_{12} \otimes y_{12} \otimes y_{12} \otimes y_{12} \otimes z_{15}+a
$$


where $a=\sum a_{1} \otimes \cdots \otimes a_{5}$ such that $a_{i}=z_{15}$ for some $i \leq 4$ or $a_{i}=1$ for at least one and at most four $i$ 's and $a_{j} \in \widehat{K(2)_{*}}\left(E_{8(5)}\right)\left(=\widehat{K(2)_{*}}\left(E_{8}\right)\right)$ for all $j$ with $a_{j} \neq 1$. Since $c_{5 *}(g \times g \times g \times g \times i d)_{*}(a)=0$ by 2.3 and (2.3), it follows from 2.1 that we have

$$
\left[g,[g,[g,[g, i d]]]_{*}\left(y_{12}^{4} z_{15}\right)=24 \operatorname{ad}^{4}\left(y_{12}\right)\left(z_{15}\right)=-\operatorname{ad}^{4}\left(y_{12}\right)\left(z_{15}\right) \neq 0\right.
$$

in $K(2)_{*}\left(E_{8}\right)$ and $\operatorname{nil}\left[E_{8(5)}, E_{8(5)}\right] \geq 5$ so that $\operatorname{nil}\left[E_{8}, E_{8}\right] \geq 5$ by $(2.1)$.

Proposition 2.5. $\operatorname{nil}[\operatorname{Spin}(8), \operatorname{Spin}(8)] \geq \operatorname{nil}[\operatorname{Spin}(7), \operatorname{Spin}(7)] \geq 3$.

Proof. Since the bundle $\operatorname{Spin}(7) \rightarrow \operatorname{Spin}(7) / G_{2}=S^{7}$ has a 3 section, there is a mod 2 equivalence $\operatorname{Spin}(7) \simeq_{2} S^{7} \times G_{2}$. In particular the inclusion $i_{(2)}: G_{2(2)} \rightarrow$ $\operatorname{Spin}(7)_{(2)}$ has a homotopy left inverse. Thus the following homomorphism $i_{(2) *}$ is injective and $i_{(2)}^{*}$ is surjective:

$$
\left[\operatorname{Spin}(7)_{(2)}, \operatorname{Spin}(7)_{(2)}\right] \stackrel{i_{(2) *}}{\longleftarrow}\left[\operatorname{Spin}(7)_{(2)}, G_{2(2)}\right] \stackrel{i_{(2)}^{*}}{\longrightarrow}\left[G_{2(2)}, G_{2(2)}\right] .
$$

Hence

$$
\operatorname{nil}\left[\operatorname{Spin}(7)_{(2)}, \operatorname{Spin}(7)_{(2)}\right] \geq \operatorname{nil}\left[\operatorname{Spin}(7)_{(2)}, G_{2(2)}\right] \geq \operatorname{nil}\left[G_{2(2)}, G_{2(2)}\right] .
$$

Since the localization is an exact functor, it follows from Theorem 2.3 of [13] that

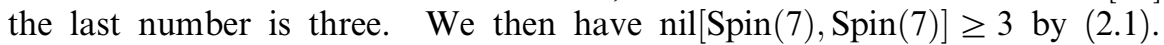

Since the bundle $\operatorname{Spin}(8) \rightarrow \operatorname{Spin}(8) / \operatorname{Spin}(7)=S^{7}$ has a section, there is a homeomorphism $\operatorname{Spin}(8) \approx S^{7} \times \operatorname{Spin}(7)$. In particular the inclusion $i: \operatorname{Spin}(7) \subset \operatorname{Spin}(8)$ has a left inverse. By the same method as above, we have $\operatorname{nil}[\operatorname{Spin}(8), \operatorname{Spin}(8)] \geq \operatorname{nil}[\operatorname{Spin}(7), \operatorname{Spin}(7)]$. This completes the proof.

Remark 2.6. By using mod 2 versions of 2.2 and 2.3, we can prove noncommutativity of $[\operatorname{Spin}(7), \operatorname{Spin}(7)]$.

\section{3. $S U(4)$}

The purpose of this section is to prove

Proposition 3.1. $\operatorname{nil}[S U(4), S U(4)]=3$.

We have nil[SU(4), $S U(4)] \leq$ cat $S U(4)=3$ by (1.1) and [15]. It then suffices to show the existence of three maps $a_{1}, a_{2}, a_{3}: S U(4) \rightarrow S U(4)$ such that $\left[a_{1},\left[a_{2}, a_{3}\right]\right] \neq 0$.

Let $p: S U(4) \rightarrow S^{7}, p: S U(3) \rightarrow S^{5}, p^{\prime}: S U(4) \rightarrow S U(4) / S p(2)=S^{5}$ be the canonical projections, $\theta: S^{3}=S U(2) \rightarrow S U(n)(n \geq 3), i: S U(3) \rightarrow S U(4)$ the inclusions, and $l_{n}$ the identity map of $S^{n}$.

Recall from [1], [10] the following: 


$$
\begin{aligned}
& \pi_{5}(S U(3))=\mathrm{Z}\{[2]\} \stackrel{i_{*}}{\cong} \pi_{5}(S U(4)), \quad p_{*}[2]=2 l_{5}, \\
& \pi_{7}(S U(4))=\mathrm{Z}\{[6]\}, \quad p_{*}[6]=6 l_{7}, \quad p_{*}^{\prime}: \pi_{8}(S U(4)) \cong \pi_{8}\left(S^{5}\right)=\mathrm{Z}_{24}, \\
& \pi_{12}(S U(3)) \stackrel{i_{*}}{\cong} \pi_{12}(S U(4))=\mathrm{Z}_{60}\left\{\left\langle i_{*}[2],[6]\right\rangle\right\}, \\
& \pi_{15}(S U(3))=\mathrm{Z}_{36} \stackrel{i_{*}}{\complement} \pi_{15}(S U(4))=\mathrm{Z}_{72} \oplus \mathrm{Z}_{2} .
\end{aligned}
$$

There exists a map $g$ which makes the following diagram commutative up to homotopy:

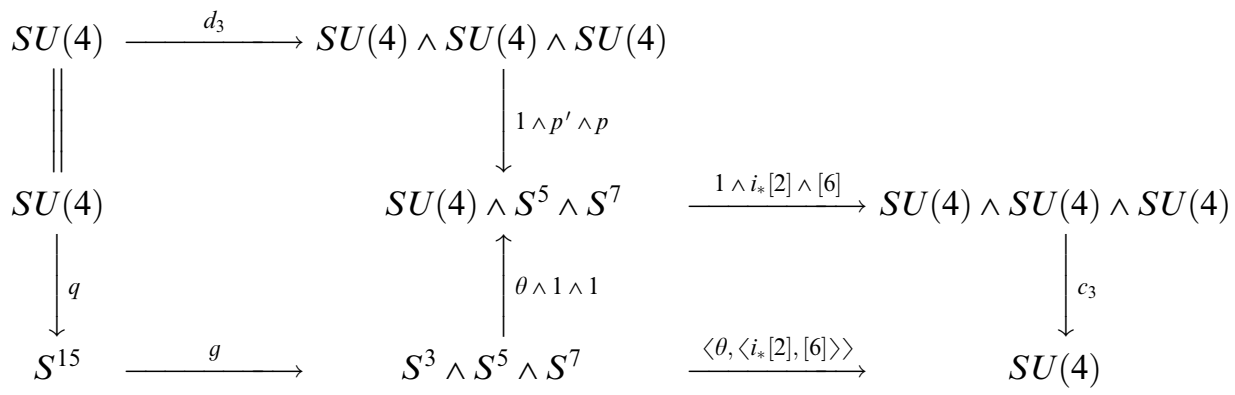

By using integral cohomology, we see that $g$ is a homotopy equivalence. Hence

$$
\left[1,\left[i \circ[2] \circ p^{\prime},[6] \circ p\right]\right]= \pm q^{*}\left\langle\theta,\left\langle i_{*}[2],[6]\right\rangle\right\rangle .
$$

We shall prove non-triviality of these elements. Let $\eta_{2}: S^{3} \rightarrow S^{2}$ be the Hopf map and write $\eta_{n}=\Sigma^{n-2} \eta_{2}$. Then $\pi_{n+1}\left(S^{n}\right)=Z_{2}\left\{\eta_{n}\right\}$ for $n \geq 3$ by [16]. There is a cell-decomposition:

$$
S U(4)=S^{3} \cup_{\eta_{3}} e^{5} \cup e^{8} \cup e^{7} \cup e^{10} \cup e^{12} \cup_{\xi} e^{15} .
$$

We have an exact sequence:

$$
\left[\Sigma S U(4)^{(14)}, S U(4)\right] \stackrel{\Sigma \xi^{*}}{\longrightarrow} \pi_{15}(S U(4)) \stackrel{q^{*}}{\longrightarrow}[S U(4), S U(4)]
$$

where $X^{(k)}$ denotes the $k$-skeleton of a CW-complex $X$. The following implies that the order of $\left[1,\left[i \circ[2] \circ p^{\prime},[6] \circ p\right]\right]$ is a multiple of three so that 3.1 follows.

Lemma 3.2. (1) The order of $\left\langle\theta,\left\langle i_{*}[2],[6]\right\rangle\right\rangle$ is a multiple of three.

(2) $2^{7}\left[\Sigma S U(4)^{(14)}, S U(4)\right]=0$.

Proof. (1) Let $\beta \in \pi_{12}(S U(3))$ be a generator. Then, from (3.1) and (3.2), it suffices to show that the order of $\langle\theta, \beta\rangle \in \pi_{15}(S U(3))$ is a multiple of three. By (15.14) of [5], we have

$$
p_{*}\langle\theta, \beta\rangle=\left\langle\iota_{3}, p_{*} \beta\right\rangle_{r}
$$

where $\langle,\rangle_{r}: \pi_{s}\left(S^{3}\right) \times \pi_{t}\left(S U(3) / S^{3}\right) \rightarrow \pi_{s+t}\left(S U(3) / S^{3}\right)$ is the relative Samelson product. It follows from [10], [16] that $p_{*}: \pi_{12}(S U(3)) \rightarrow \pi_{12}\left(S^{5}\right)=\mathrm{Z}_{3}\left\{\alpha_{2}(5)\right\}$ 
$\oplus \mathrm{Z}_{2} \oplus \mathrm{Z}_{5} \quad$ is surjective, $\pi_{15}\left(S^{8}\right)=\mathrm{Z}_{3}\left\{\alpha_{2}(8)\right\} \oplus \mathrm{Z} \oplus \mathrm{Z}_{8} \oplus \mathrm{Z}_{5}$, and $\pi_{15}\left(S^{5}\right)=$ $\mathrm{Z}_{9}\left\{\beta_{1}(5)\right\} \oplus \mathrm{Z}_{8} \oplus \mathrm{Z}_{2}, 3 \beta_{1}(5)=-\alpha_{1}(5) \circ \alpha_{2}(8)$. Also by (16.2) of [5], we have $\left\langle l_{3}, l_{5}\right\rangle_{r}=J_{\mathrm{C}}\left(l_{3}\right)$, where $J_{\mathrm{C}}: \pi_{3}(S U(2)) \rightarrow \pi_{3}(S O(4)) \stackrel{J}{\rightarrow} \pi_{7}\left(S^{4}\right) \stackrel{\Sigma}{\rightarrow} \pi_{8}\left(S^{5}\right)$ is the complex $J$-homomorphism. Since $J_{\mathrm{C}}$ is surjective in this case, we have

$$
\left\langle l_{3}, l_{5}\right\rangle_{r} \in \pi_{8}\left(S^{5}\right)=\mathrm{Z}_{8} \oplus \mathrm{Z}_{3}\left\{\alpha_{1}(5)\right\} \quad \text { is a generator. }
$$

It follows from (16.5) of [5] that $\left\langle l_{3}, \Sigma x\right\rangle_{r}=\left\langle l_{3}, l_{5}\right\rangle_{r} \circ \Sigma^{4} x$ for any $x \in \pi_{m}\left(S^{4}\right)$. Hence $\left\langle l_{3}, \alpha_{2}(5)\right\rangle_{r}=\left\langle l_{3}, l_{5}\right\rangle_{r} \circ \alpha_{2}(8)= \pm \alpha_{1}(5) \circ \alpha_{2}(8)= \pm 3 \beta_{1}(5) \neq 0$ by (3.4). Therefore the 3 -component of $\left\langle l_{3}, p_{*} \beta\right\rangle_{r}$ is $\pm 3 \beta_{1}(5)$. Since $p_{*}: \pi_{15}(S U(3)) \rightarrow$ $\pi_{15}\left(S^{5}\right)$ is injective by [10], it follows from (3.3) that the order of $\langle\theta, \beta\rangle$ is a multiple of three.

(2) Let $\gamma: S^{5} \rightarrow \mathrm{C} P^{2}$ be the canonical map. Then $S U(4)^{(7)}=\Sigma \mathrm{C} P^{3}=$ $S^{3} \cup_{\eta_{3}} e^{6} \cup_{\Sigma \gamma} e^{7}$. By Proposition 1.15 of [11], $\Sigma^{3} \gamma$ is homotopic to the composite of $S^{8} \stackrel{2 g}{\longrightarrow} S^{5} \stackrel{j}{\subset} \Sigma^{3} \mathrm{C} P^{2}$, where $g \in \pi_{8}\left(S^{5}\right)$ is a generator. Write $A_{4}=$ $\left[\Sigma S U(4)^{(7)}, S U(4)\right]$. There is a commutative diagram:

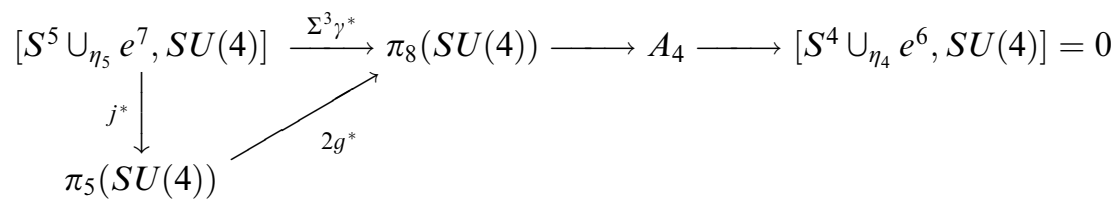

Since $\pi_{6}(S U(4))=0, \quad j^{*}$ is surjective and $p_{*}^{\prime}(2 g)^{*} i_{*}[2]=2 l_{5} \circ 2 g=4 g$. Hence $\operatorname{Im}\left(\Sigma^{3} \gamma^{*}\right)=4 \pi_{8}(S U(4))$ and $A_{4}=Z_{4}$. Write $A_{1}=\left[\Sigma S U(4)^{(14)}, S U(4)\right], A_{2}=$ $\left[\Sigma S U(4)^{(10)}, S U(4)\right]$, and $A_{3}=\left[\Sigma S U(4)^{(8)}, S U(4)\right]$. The following diagram implies that $2^{7} A_{1}=0$ :

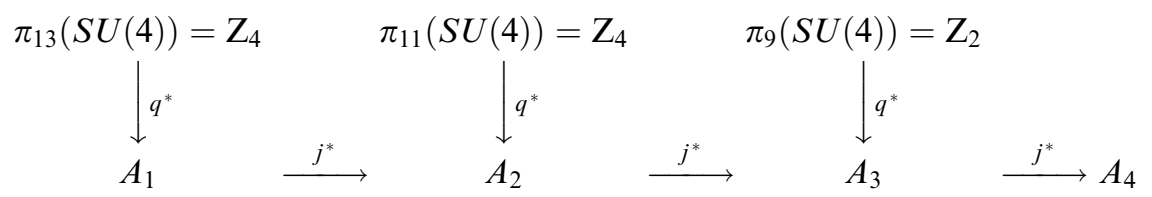

4. $S U(5), S U(6), S p(3)$

Let $(G, d)$ be $(S U, 2)$ or $(S p, 4)$. Let $\theta: S^{3} \subset G(n)$ be the inclusion map and $\alpha \in \pi_{d n-1}(G(n))=\mathrm{Z}$ a generator. We refer to [8] for homotopy groups of Lie groups.

THeOREM 4.1 ([1]). The order of the Samelson product $\langle\theta, \alpha\rangle \in \pi_{d n+2}(G(n))$ is

$$
\begin{cases}n(n+1) & (G=S U \text { and } n \geq 3) \\ n(2 n+1) \varepsilon_{n} & (G=S p \text { and } n \geq 2)\end{cases}
$$

where $\varepsilon_{n}$ is 1 or 4 according as $n$ is even or odd.

Proof. The case of $\operatorname{Sp}(n)$ follows from Theorem 2 of [1]. By Theorem 1 
of [1], the order of $j_{*}\langle\theta, \alpha\rangle \in \pi_{2 n+2}(S U(n+1))$ is $n(n+1)$ for $n \geq 2$, where $j: S U(n) \subset S U(n+1)$. It then follows from the structure of $j_{*}: \pi_{2 n+2}(S U(n))$

$\rightarrow \pi_{2 n+2}(S U(n+1))$ (see [8]) that the order of $\langle\theta, \alpha\rangle$ is $n(n+1)$ for $n \geq 3$.

Recall that $G(n)$ has a cell-decomposition:

$$
G(n)=G(n-1) \cup e^{d n-1} \cup_{\rho_{n}} e^{d n+2} \cup\{\text { cells of dimension } \geq d(n+1)+2\} .
$$

We use always this decomposition. Write $Y(n)=G(n-1)^{(d n+1)} \cup e^{d n-1}$ and $Z(n)=Y(n) \cup_{\rho_{n}} e^{d n+2}$. Let $p: G(n) \rightarrow S^{d n-1}$ be the canonical projection. For simplicity we denote by 1 the identity maps, by $j$ the inclusion maps, and by $q$ the quotient maps. There exists a map $g$ which makes the following diagram commutative up to homotopy:

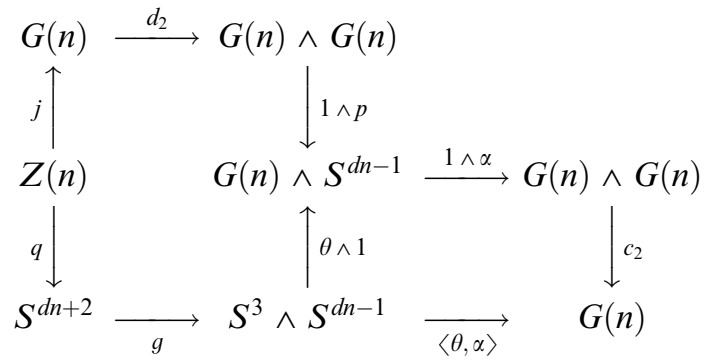

By using the integral cohomology, we have that $g$ is a homotopy equivalence so that

$$
j^{*}[1, \alpha \circ p]= \pm q^{*}\langle\theta, \alpha\rangle .
$$

If these elements are non-zero, then $[1, \alpha \circ p] \neq 0$ and $[G(n), G(n)]$ is noncommutative. To study non-triviality of $q^{*}\langle\theta, \alpha\rangle$, we compare the orders of $\langle\theta, \alpha\rangle$ and the image of $\Sigma \rho_{n}^{*}$ :

$$
[\Sigma Y(n), G(n)] \stackrel{\Sigma \rho_{n}^{*}}{\longrightarrow} \pi_{d n+2}(G(n)) \stackrel{q^{*}}{\longrightarrow}[Z(n), G(n)] .
$$

Consider the following commutative diagram:

$$
\begin{aligned}
& \pi_{d n+2}\left(\Sigma G(n-1)^{(d n+1)}\right) \stackrel{j_{*}}{\longrightarrow} \pi_{d n+2}(\Sigma Y(n)) \longrightarrow \pi_{d n+2}\left(\Sigma Y(n), \Sigma G(n-1)^{(d n+1)}\right) \\
& j^{j_{*}} \quad \cong \downarrow q_{*} \\
& \pi_{d n+2}(\Sigma G(n)) \underset{\Sigma p_{*}}{\longrightarrow} \quad \pi_{d n+2}\left(S^{d n}\right)
\end{aligned}
$$

Since $j_{*}\left(\Sigma \rho_{n}\right)=0$, there exists $\tilde{\rho}_{n} \in \pi_{d n+2}\left(\Sigma G(n-1)^{(d n+1)}\right)$ such that $j_{*}\left(\tilde{\rho}_{n}\right)=\Sigma \rho_{n}$. Hence $\Sigma \rho_{n}^{*}$ in (4.1) decomposes as

$$
[\Sigma Y(n), G(n)] \stackrel{j^{*}}{\longrightarrow}\left[\Sigma G(n-1)^{(d n+1)}, G(n)\right] \stackrel{\tilde{\rho}_{n}^{*}}{\longrightarrow} \pi_{d n+2}(G(n))
$$

and so we have 


$$
\operatorname{Im}\left(\Sigma \rho_{n}^{*}\right) \subset \operatorname{Im}\left(\tilde{\rho}_{n}^{*}\right) .
$$

We can show that $j^{*}$ of (4.2) is surjective so that $\operatorname{Im}\left(\Sigma \rho_{n}^{*}\right)=\operatorname{Im}\left(\tilde{\rho}_{n}^{*}\right)$. But we do not use this.

Problem 4.2. Is there a prime $p$ satisfying $v_{p}\left(\sharp \operatorname{Im}\left(\tilde{\rho}_{n}^{*}\right)\right)<v_{p}(\sharp\langle\theta, \alpha\rangle)$ ?

Here $\sharp$ denotes the order and $v_{p}(m)$ is the exponent of $p$ in the prime decomposition of an integer $m$. Notice that $q^{*}\langle\theta, \alpha\rangle$ is non-zero if Problem 4.2 is affirmative.

Proposition 4.3. Problem 4.2 is affirmative when $G(n)$ is one of the following:

$$
S p(2), S p(3), S U(3), S U(4), S U(5), S U(6) .
$$

Proof. It is easy to show the following: $\tilde{\rho}^{*}=0$ for $S p(2), S U(3)$ and $2 \cdot \tilde{\rho}^{*}=0$ for $S U(4)$. Hence the result follows from 4.1 for these cases. We omit the details.

$S p(3)$. Consider the following exact sequence:

$$
\pi_{11}(\operatorname{Sp}(3)) \stackrel{q^{*}}{\longrightarrow}\left[\Sigma S p(2)^{(13)}, S p(3)\right] \stackrel{j^{*}}{\longrightarrow}\left[S^{4} \cup_{\eta} e^{8}, S p(3)\right]
$$

Here $S p(2)^{(13)}=S p(2)=S^{3} \cup e^{7} \cup e^{10}$. We have $j^{*}:\left[S^{4} \cup e^{8}, S p(3)\right] \cong \pi_{4}(S p(3))$ $=\mathrm{Z}_{2}$ by $[8]$. Hence $2\left[\Sigma \operatorname{Sp}(2)^{(13)}, \operatorname{Sp}(3)\right] \subset \operatorname{Im}\left(q^{*}\right)$ and so $2 \cdot \operatorname{Im}\left(\tilde{\rho}^{*}\right) \subset \operatorname{Im}(q \circ \tilde{\rho})^{*}$. Hence $48 \cdot \operatorname{Im}\left(\tilde{\rho}^{*}\right) \subset 24 \cdot \operatorname{Im}(q \circ \tilde{\rho})^{*}=0$, since $q \circ \tilde{\rho} \in \pi_{14}\left(S^{11}\right)=Z_{24}$. Therefore $v_{7}\left(\sharp \operatorname{Im}\left(\tilde{\rho}^{*}\right)\right)=0<v_{7}(\sharp\langle\theta, \alpha\rangle)=1$.

Let $n$ be 5 or 6 . Then, we have $S U(n-1)^{(2 n+1)}=S U(n-1)^{(2 n)}$ and $S U(n-1)^{(2 n-1)}=S U(n-1)^{(2 n-2)}=S U(n-2)^{(2 n-2)} \cup e^{2 n-3}$. Since $2 \pi_{2 n+2}$ $\left(\Sigma S U(n-1)^{(2 n)}, \Sigma S U(n-1)^{(2 n-2)}\right)=0$, there exists $\hat{\rho} \in \pi_{2 n+2}\left(\Sigma S U(n-1)^{(2 n-2)}\right)$ such that $j_{*} \hat{\rho}=2 \tilde{\rho} \in \pi_{2 n+2}\left(\Sigma S U(n-1)^{(2 n)}\right)$. By Theorem (2.1) of [4], we have $\pi_{2 n+2}\left(\Sigma S U(n-1)^{(2 n-2)}, \Sigma S U(n-2)^{(2 n-2)}\right)=0$. Hence there exists $\bar{\rho} \in \pi_{2 n+2}$ $\left(\Sigma S U(n-2)^{(2 n-2)}\right)$ such that $j_{*} \bar{\rho}=\hat{\rho} \in \pi_{2 n+2}\left(\Sigma S U(n-1)^{(2 n-2)}\right)$. Thus

$$
2 \cdot \operatorname{Im}\left(\tilde{\rho}^{*}\right) \subset \operatorname{Im}\left\{\bar{\rho}^{*}:\left[\Sigma S U(n-2)^{(2 n-2)}, S U(n)\right] \rightarrow \pi_{2 n+2}(S U(n))\right\} .
$$

Let $n=5$ in (4.3). Since $\left[S^{4} \cup e^{6}, S U(5)\right]=0, q^{*}: \pi_{9}(S U(5)) \rightarrow[\Sigma S U(3)$, $S U(5)]$ is surjective. Hence $\operatorname{Im}\left(\bar{\rho}^{*}\right)=\operatorname{Im}(q \circ \bar{\rho})^{*}$. Since $q \circ \bar{\rho} \in \pi_{12}\left(S^{9}\right)=\mathrm{Z}_{24}$, $24 \cdot \operatorname{Im}\left(\bar{\rho}^{*}\right)=0$ and $48 \cdot \operatorname{Im}\left(\tilde{\rho}^{*}\right)=0$ by (4.3). Thus $v_{5}\left(\sharp \operatorname{Im}\left(\tilde{\rho}^{*}\right)\right)=0<v_{5}(\sharp\langle\theta, \alpha\rangle)$ $=1$.

Let $n=6$ in (4.3). First we prove

$$
\left[\Sigma S U(4)^{(8)}, S U(6)\right] \stackrel{j^{*}}{\cong}[\Sigma S U(3), S U(6)] \stackrel{q^{*}}{\cong} \pi_{9}(S U(6))=\mathrm{Z} .
$$

Since $\pi_{4}(S U(6))=\pi_{6}(S U(6))=\left[S^{4} \cup_{\eta_{4}} e^{6}, S U(6)\right]=0$, there are exact sequences: 


$$
\begin{aligned}
& {\left[S^{5} \cup_{\eta_{5}} e^{7}, S U(6)\right] \stackrel{\Sigma^{2} \rho_{3}^{*}}{\longrightarrow} \pi_{9}(S U(6))=\mathrm{Z} \stackrel{q^{*}}{\longrightarrow}[\Sigma S U(3), S U(6)] \longrightarrow 0} \\
& \pi_{7}(S U(6)) \stackrel{q^{*}}{\longrightarrow}\left[S^{5} \cup_{\eta_{5}} e^{7}, S U(6)\right] \stackrel{j^{*}}{\longrightarrow} \pi_{5}(S U(6)) \longrightarrow 0 .
\end{aligned}
$$

Since the attaching map of the top cell of any Lie group is stably trivial, the composite $S^{7} \stackrel{\rho_{3}}{\longrightarrow} S^{3} \cup_{\eta_{3}} e^{5} \stackrel{q}{\longrightarrow} S^{5}$ is null-homotopic. Hence $\Sigma^{2} \rho_{3}^{*}\left(\operatorname{Im}\left(q^{*}\right)\right)=0$. Since $\pi_{6}(S U(4))=0$, the map $S^{5} \stackrel{|2|}{\longrightarrow} S U(3) \subset S U(4)$ can be extended to a map $f: S^{5} \cup_{\eta_{5}} e^{7} \rightarrow S U(4)$. Write $\tilde{f}=j \circ f: S^{5} \cup_{\eta_{5}} e^{7} \rightarrow S U(6)$. Since $\Sigma^{2} \rho_{3}^{*}(f) \in$ $\pi_{9}(S U(4))=\mathrm{Z}_{2_{\tilde{f}}}$ and $\pi_{9}(S U(6))=\mathrm{Z}$, it follows that $\Sigma^{2} \rho_{3}^{*}(\tilde{f})=0$. Thus $\Sigma^{2} \rho_{3}^{*}=0$ in (4.5), since $\tilde{f}$ and $\operatorname{Im}\left(q^{*}\right)$ generate $\left[S^{5} \cup_{\eta_{5}} e^{7}, S U(6)\right]$. Therefore

$$
q^{*}: \pi_{9}(S U(6)) \cong[\Sigma S U(3), S U(6)]
$$

Since $\pi_{8}(S U(6))=0$, we have an exact sequence:

$$
0 \longrightarrow\left[\Sigma S U(4)^{(8)}, S U(6)\right] \stackrel{j^{*}}{\longrightarrow}[\Sigma S U(3), S U(6)] \stackrel{\Sigma \tau^{*}}{\longrightarrow} \pi_{7}(S U(6))=\mathrm{Z}
$$

where $\tau: S^{6} \rightarrow S U(3)$ is the attaching map of the 7-dimensional cell of $S U(4)$. Since $q \circ \Sigma \tau=0$, (4.6) implies that $\Sigma \tau^{*}=0$ so that $j^{*}$ is an isomorphism. This ends the proof of (4.4).

Since $\pi_{10}(S U(6))=0$, we have an exact sequence:

$$
\pi_{11}(S U(6)) \stackrel{q^{*}}{\longrightarrow}\left[\Sigma S U(4)^{(10)}, S U(6)\right] \stackrel{\Sigma j^{*}}{\longrightarrow}\left[\Sigma S U(4)^{(8)}, S U(6)\right] \longrightarrow 0
$$

Since $q \circ \bar{\rho} \in \pi_{14}\left(S^{11}\right)=\mathrm{Z}_{24}$, we have

$$
24 \cdot \bar{\rho}^{*}\left(\operatorname{Im}\left(q^{*}\right)\right)=0 .
$$

Let $h \in\left[\Sigma S U(4)^{(10)}, S U(6)\right]$ be such that $\bar{h}:=\Sigma j^{*} h$ is the composite of

$$
\Sigma S U(4)^{(8)} \stackrel{q}{\longrightarrow} S^{9} \stackrel{[4 !]}{\longrightarrow} S U(5) \stackrel{\subset}{\longrightarrow} S U(6)
$$

where [4!] is a generator of $\pi_{9}(S U(5))=\mathrm{Z}$. By (4.4), $\bar{h}$ is a generator of $\left[\Sigma S U(4)^{(8)}, S U(6)\right]=Z$. Let $\omega: S^{9} \rightarrow S U(4)^{(8)}$ be the attaching map of the unique 10-dimensional cell of $S U(4)$. Since $q \circ \Sigma \omega \in \pi_{10}\left(S^{9}\right)=Z_{2}$, there exists $k: \Sigma S U(4)^{(10)} \rightarrow S^{9}$ such that the following diagram is commutative up to homotopy:

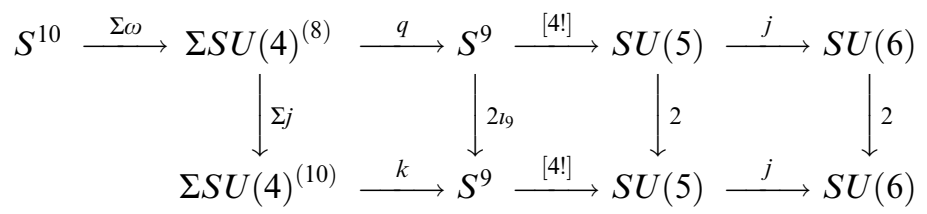

where the latter two 2's are power maps $x \mapsto x^{2}$. We then have $(\Sigma j)^{*}(j \circ[4 !] \circ k)$ $=2 \bar{h}=(\Sigma j)^{*}(2 h)$ and

$$
2 h-j \circ[4 !] \circ k \in \operatorname{Im}\left\{q^{*}: \pi_{11}(S U(6)) \rightarrow\left[\Sigma S U(4)^{(10)}, S U(6)\right]\right\} .
$$


Since $k \circ \bar{\rho} \in \pi_{14}\left(S^{9}\right)=0$, we have $2 \cdot \bar{\rho}^{*}(h) \in \bar{\rho}^{*}\left(\operatorname{Im}\left(q^{*}\right)\right)$. Thus $48 \cdot \bar{\rho}^{*}(h)=0$ by (4.7), and hence $48 \cdot \operatorname{Im}\left(\bar{\rho}^{*}\right)=0$. Therefore $96 \cdot \operatorname{Im}\left(\tilde{\rho}^{*}\right)=0$ by (4.3). Hence $v_{7}(\sharp \operatorname{Im}(\tilde{\rho}))=0<v_{7}(\sharp\langle\theta, \alpha\rangle)=1$. This completes the proof.

\section{REFERENCES}

[1] R. Botт, A note on the Samelson product in the classical groups, Comment. Math. Helv., 34 (1960), 249-256.

[2] J. Harper, The mod 3 homotopy type of $F_{4}$, Lecture Notes in Math. 418, Springer, 1974, $58-67$.

[3] B. Harris, Suspensions and characteristic maps for symmetric spaces, Ann. of Math., 76 (1962), 295-305.

[ 4 ] I. M. JAMES, On the homotopy groups of certain pairs and triads, Quart. J. Math., 5 (1954), 260-270.

[ 5] I. M. James, The Topology of Stiefel Manifolds, London Math. Soc. Lecture Note Series 24, Cambridge, 1976.

[ 6 ] K. Kudou and N. Yagita, Modulo odd prime homotopy normality for $H$-spaces, J. Math. Kyoto Univ., 38 (1998), 643-651.

[ 7 ] K. Kudou and N. Yagita, Highly homotopy non-commutativity of Lie groups with 2-torsion, Kyushu J. Math., 53 (1999), 133-150.

[ 8 ] M. Mimura, Homotopy theory of Lie groups, Handbook of Algebraic Topology (I. M. James ed.), Elsevier, 1995, 951-991.

[9] M. Mimura and H. Ōshima, Self homotopy groups of Hopf spaces with at most three cells, J. Math. Soc. Japan, 51 (1999), 71-92.

[10] M. Mimura and H. Toda, Homotopy groups of $S U(3), S U(4)$ and $S p(2)$, J. Math. Kyoto Univ., 3 (1964), 217-250.

[11] H. Ōshima, On stable James numbers of stunted complex or quaternionic projective spaces, Osaka J. Math., 16 (1979), 479-504.

[12] H. Ōshima, Self homotopy set of a Hopf space, Quart. J. Math., 50 (1999), 483-495.

[13] H. Ōshima, Self homotopy group of the exceptional Lie group $G_{2}$, J. Math. Kyoto Univ., 40 (2000), 177-184.

[14] V. R. RAo, $\operatorname{Spin}(n)$ is not homotopy nilpotent for $n \geq 7$, Topology, 32 (1993), 239-249.

[15] W. Singhof, On the Lusternik-Schnirelmann category of Lie groups, Math. Z., 145 (1975), $111-116$.

[16] H. Toda, Composition Methods in Homotopy Groups of Spheres, Ann. of Math. Study 49, Princeton 1962.

[17] G. W. Whitehead, Elements of Homotopy Theory, Graduate Texts in Math. 61, Springer, 1978.

[18] C. WiLKerson, Self maps of classifying spaces, Lecture Notes in Math. 418, Springer, 1974, 150-157.

[19] N. YaGita, Homotopy nilpotency for simply connected Lie groups, Bull. London Math. Soc., 25 (1993), 481-486.

[20] N. Yagita, Pontrjagin rings of the Morava K-theory for finite H-spaces, J. Math. Kyoto Univ., 36 (1996), 447-452.

IBARAKI UNIVERSITY

Mito, IBARAKI, 310-8512

JAPAN

E-mail: ooshima@mito.ipc.ibaraki.ac.jp

yagita@mito.ipc.ibaraki.ac.jp 